\title{
A STUDY ON THE BIOEQUIVALENCE OF LITHIUM AND VALPROATE SALIVARY AND BLOOD LEVELS IN THE TREATMENT OF BIPOLAR DISORDER
}

Murru $\mathrm{A}^{\mathrm{a}}$, Torra $\mathrm{M}^{\mathrm{b}}$, Callari $\mathrm{A}^{\mathrm{c}}$, Pacchiarotti $\mathrm{I}^{\mathrm{a}}$, Romero $\mathrm{S}^{\mathrm{d}}$, Gonzalez de la Presa $\mathrm{B}^{\mathrm{e}}$, Varo $\mathrm{C}^{\mathrm{a}}$, Goikolea $\mathrm{JM}^{\mathrm{a}}$, Pérez-Sola $\mathrm{V}^{\mathrm{f}}$, Vieta $\mathrm{E}^{\mathrm{a}}$, Colom $\mathrm{F}^{\mathrm{f} *}$

a) Bipolar Disorders Unit, Institute of Neuroscience, Hospital Clínic, University of Barcelona, IDIBAPS, CIBERSAM, Villarroel 170, Barcelona 08036, Catalonia, Spain.

b) Pharmacology and Toxicology, Biomedical Diagnosis Department, Hospital Clínic, University of Barcelona, IDIBAPS, Villarroel 170, Barcelona 08036, Catalonia, Spain.

c) Department of Clinical and Experimental Medicine, Section of Psychiatry, University of Pisa, Lungarno Pacinotti 44 I-56126, Pisa, Italy.

d) Department of Child and Adolescent Psychiatry and Psychology, Institute of Neuroscience, CIBERSAM, Hospital Clínic of Barcelona, Villarroel 170, Barcelona 08036 ,Catalonia, Spain.

e) CORE Laboratory- Centre de Diagnòstic Biomédic Hospital Clinic, IDIBAPS, Villarroel 170, Barcelona 08036, Catalonia Spain.

f) Mental Health Group, IMIM Hospital del Mar, CIBERSAM, Plaza Charles Darwin, sn, 08003 Barcelona, Catalonia, Spain.

* Corresponding author:

Dr. Francesc Colom,

Mental Health Group,

IMIM Hospital del Mar,

CIBERSAM

Plaza Charles Darwin, sn, 08036 Barcelona (ES)

Tel: +34933160624

Fax: +34933160410

Email: fcolom@imim.es 


\section{ABSTRACT}

Lithium (Li) and valproate (VPA) are commonly used in the treatment of bipolar disorder (BD), with narrow therapeutic window requiring periodic control of serum levels. This prevents intoxication, lack of efficacy due to low serum concentrations, and allows monitoring treatment adherence. We aimed at evaluating the bioequivalence of salivary and blood levels of LI or VPA in a sample of adult BD patients. Secondarily, lithium bioequivalence was evaluated across different patients' lifespans. BD patients treated with either Li or VPA underwent contemporary standard serum and salivary measurements. Blood levels of both drugs were taken according to standard procedures. Li salivary levels were performed by an adapted potentiometric method on the AVL9180 electrolyte analyzer and confirmed by means of a Perkin-Elmer AA200 flame atomic absorption spectrometer. VPA salivary levels were taken with an immune-assay method with turbidimetric inhibition. Correlations were calculated with Pearson Product Moment Correlation in an interim analysis. Age was controlled with partial correlation technique. A total of 50 patients (38 on Li, 12 on VPA) were enrolled. Blood-saliva bioequivalence for VPA was not found due to a high variability in salivary measures. Li measures resulted in a high correlation ( $\mathrm{r}=0.767$, $\mathrm{p}<0.001)$, showing no partial correlation with age $(\mathrm{r}=0.147, \mathrm{p}=0.380)$. Li salivary test is a reliable method of measuring Li availability and is equivalent to serum levels. Potential advantages of Li salivary testing are its non-invasive nature and the possibility of doing the test during the usual appointment with the psychiatrist. 


\section{INTRODUCTION}

Bipolar Disorder (BD) is a highly prevalent and chronic condition, with high risk of relapses into acute episodes within 5 years (Keller et al., 1993; Merikangas et al., 2011). For this reason, an appropriate longterm treatment with mood stabilizers aimed at preventing recurrences is necessary (Grande et al., 2015).

In the last twenty years several drugs for the treatment of acute phases and for the prevention of relapses in BD have been introduced (Carvalho et al., 2015). Along with newer agents, "classical" mood stabilizers such as lithium (Li) and valproate (VPA) are still widely used, alone or in combination, in the long term treatment of BD (Samalin et al., 2016). Lithium still represents a cornerstone in the treatment of $\mathrm{BD}$, and the long clinical experience associated with the use of this drug makes it a relatively safe tool to prevent recurrences of BD (Geddes and Miklowitz, 2013; Nivoli et al., 2010). Valproate is also considered by most clinical guidelines as a first-line option in the maintenance phase, although some concerns on tolerability have been raised (Murru et al., 2015; Yatham et al., 2013). As both lithium and valproate have a relatively narrow therapeutic window, therapeutic ranges need to be routinely checked. Blood sampling and subsequent measuring - generally twice a year- are the methods used to check for $\mathrm{Li}$ and VPA levels in everyday clinical practice (NICE, 2014).

Unfortunately, Li has a high discontinuation rate, with an average time of adherence as short as 76 days at 6-year follow-up (Johnson and McFarland, 1996). To date there are no similar data for VPA. Poor adherence is a common issue in BD patients, with rates of 20-40\% (Colom et al., 2000; Murru et al., 2013; Sajatovic et al., 2009) in euthymic patients, and up to 40-64\% among acute manic patients (Keck et al., 1997; Montoya et al., 2007). Poorly adherent BD patients suffer from greater chronicity, worse functioning, an increased number of visits to emergency units and increased and longer acute admissions, with subsequent higher health expenses (Gianfrancesco et al., 2008; Lew et al., 2006). The risk of poor adherence has been documented in BD patients within the first weeks after initiating or changing treatment due to a manic or mixed relapse (González-Pinto et al., 2010). This is a delicate moment, in which poor illness awareness and need for effective treatment should lead clinicians to require a frequent monitoring of mood stabilizers in order to assess therapeutic blood levels, reduce risks of toxicity as well as ensuring treatment adherence.

The literature on the subject of salivary measures of drugs in patient populations affected by BD is almost nonexistent. Saliva was initially investigated as an alternative fluid for therapeutic monitoring of some anticonvulsants in the late 1970s until recent years on epileptic samples (Danhof and Breimer; Mucklow et al., 1981; Patsalos and Berry, 2013; Tonic-Ribarska et al., 2012), showing a low correlation for salivary/serum valproate levels (al Za'abi et al., 2003). On the other hand, the use of Li salivary monitoring instead of serum dosages has been deemed possible, but high interpersonal variability was reported, so that its possible use in clinical practice was questioned (Moody, 1999; Spencer et al., 1990). Recently, the feasibility of measuring salivary concentration of $\mathrm{Li}$ in $50 \mathrm{BD}$ patients was tested, and results did not allow for supporting saliva monitoring as a substitute of serum Li estimation (Shetty et al., 2012). Also, it is commonly known that interpersonal variability in drug volumes of distribution get increased as people get older (Mangoni and Jackson, 2004). 
The aim of the present study was to evaluate the bioequivalence of salivary and blood levels of Li and VPA in a sample of adult BD patients. Secondarily, Li bioequivalence in saliva and serum was evaluated across different patients' lifespans.

\section{EXPERIMENTAL PROCEDURES}

\subsection{Subjects and study design}

A study on the correlation between serum and salivary levels of lithium and valproate was performed in a group of BD patients who attend the Bipolar Disorder Unit of the Hospital Clínic of Barcelona.

Inclusion criteria were: 1. A diagnosis of BD, type I, II or NOS (American Psychiatric Association, 2000). 2. Age between 10 and 80 years old. 3. Being on maintenance treatment, in mono- or polytherapy, with lithium or valproate, and stable on treatment with Li or VPA for at least 4 weeks. 4 . Not with signs, symptoms or actual history suggesting ongoing dehydration. 5. Renal function within normal limits. 6. Written informed consent provided. Exclusion criteria referred to factors possibly modulating volume distribution or excretion of the studied drugs: 1. Factors affecting normal salivary secretion (active oral infections, occasional or chronic xerostomia). 2. Alcohol consumption. 3. Pregnancy. 4. Drugs affecting Li/VPA pharmacokinetics such as those significantly affecting extracellular water or kidney function. 5. Concurrent urinary tract infection.

As a secondary objective of the study was to evaluate the effect of age in saliva-blood lithium concentrations, we recruited 5 patients aged under 18 .

\subsection{Procedure}

A single visit for each patient was scheduled, in which salivary and blood sample were taken. Patients were asked to fast since the night before. All extractions were performed by a nurse. Serum levels were extracted by standard procedure. Saliva was collected by chewing parafilm wax for 1 min before spitting into tubes (Salivette ${ }^{\circledR}$ SARSTEDT). After centrifugation, the saliva samples were stored in a freezer ($20^{\circ} \mathrm{C}$ ) before analysis at the Centre de Diagnòstic Biomédic, Hospital Clinic Barcelona.

\subsection{Analytical methods}

Lithium in serum and in salivary fluid was determined by means of an electrolyte analyzer AVL 9180, using an adapted procedure based in a classic direct potentiometric method (Bertholf et al., 1988), and by means of a Perkin-Elmer AA 200 atomic absorption spectrophotometer, after appropriate dilutions with deionized water (Shetty et al., 2012). For potentiometric method, all samples of saliva were pre-treated with $\mathrm{NaCl} 0.5 \mathrm{~mol} / \mathrm{L}$ as a matrix modifier. The accuracy and precision of the results in salivary fluid were assessed by a simultaneous analysis of two pool of saliva with a mean lithium concentration of 1.2 and $2.5 \mathrm{mEq} / \mathrm{L}$. The coefficient of variation "between -day" was 0.046 and "within-run" was 0.014 . For the results in serum, the accuracy and precision were assessed by a simultaneous analysis of the standard reference material ISE-trol Quality Control Level I, II and III for Roche AVL 9180. The coefficient of 
variation between the series was 0.034 and within the series was 0.015 . The within-run imprecision $(\mathrm{CVw})$ was obtained by measuring the reference material 5 times within two hours and the interserial imprecision $(\mathrm{CVb})$ was obtained by measuring this material over a period of 10 consecutive days. The limit of detection (LOD) was $0.03 \mathrm{mEq} / \mathrm{L}$ and the limit of quantification (LOQ) was $0.1 \mathrm{mEq} / \mathrm{L}$.

Valproic acid concentration in serum is measured with a homogeneous particle enhanced turbidimetric inhibitioninmunoassay method on the Dimension EXL System. The Assay reagent, reference and provider are: VALP Dimension EXL, Ref DF78, Siemens Diagnostics (Newark, DE 19714 USA). This method has a detection limit of $3 \mathrm{mg} / \mathrm{L}$ that invalidates its use in saliva samples. In order to improve it, some modifications on the procedure were performed which involved an increase on the amount of pipetted sample and value change of the calibrators. Once these modifications were made, the new limit of detection following the recommendations of CLSI1 was estimated. This optimization method showed a notorious improvement of the assay detection limit, decreasing from 3 to $0.4 \mathrm{mg} / \mathrm{L}$.

Saliva samples were measured using the latter method

\subsection{Statistical analysis}

A sample of 20 subjects would yield $80 \%$ power for detecting an effect size of at least 0.6 using the test for dependent correlations with a significance level of 0.05 . Nonetheless, given the high dispersion of VPA measures observed, after an interim analysis it was decided to interrupt VPA patients enrollment.

Data on Li or VPA concentrations were subjected to conventional methods of statistical analysis. Correlations were calculated with Pearson Product Moment Correlation (Pearson's r) in an interim analysis. Age was controlled with partial correlation technique. Statistical analyses were performed with SPSS software (ver 19.0).

\subsection{Ethical aspects}

The protocol of the study was approved by the Hospital Ethical Committee, after a consultation to the Spanish Agency of Drugs and Health-Related Products (AEMPS). Patients had to give a signed written informed consent in order to participate in the study.

\section{RESULTS}

A total of 50 patients (38 on $\mathrm{Li}$ and 12 on VPA) were enrolled in the present cross-sectional study. Among these, 27 (54\%) were female, 46 (92\%) were diagnosed with BD type I and 4 (8\%) with BD type II. Characteristics of the sample and sample mean serum and salivary concentrations of Li and VPA are presented in tab. 1.

The concentrations of $\mathrm{Li}$ in saliva were higher than those in serum. The scatter plot of Li salivary versus serum free concentrations (Fig. 1) revealed a good linear relationship (correlation coefficient, $r=0.767, p$ $<0.001$ ) in the present sample. Lithium sample showed no partial correlation with age (age r=0.147, $\mathrm{p}=0.380$ ). Child and adolescent patients (aged 16, 12,15, 13 and 17) showed the same behavior regarding Li concentrations than adult patients. 
The scatter plot of VPA salivary versus serum free concentrations (Fig.2.) did not allow for an assessment of a blood-saliva bioequivalence due to a high variability in salivary measures $(r=0.123$, $\mathrm{p}=0.704)$.

\section{DISCUSSION}

This cross-sectional study was aimed at assessing the possible bioequivalence of Li and VPA salivary measures to their respective concentrations in serum.

High correlation was found between Li salivary and serum concentrations, coherently with previous studies in the field (Ben-Aryeh et al., 1984; El-Mallakh et al., 2004; Neu et al., 1975; Verghese et al., 1977). As human saliva has two major components, an aqueous fraction and a mucinous mucopolysaccharide fraction (Leppi and Spicer, 1966), the removal of the mucionous component from saliva by filtration in modern measurements bears an increased degree of correlation between serum and salivary Li (El-Mallakh et al., 2004). These findings seems at odds with the scant attention given to this monitoring possibility after the positive studies published in the 70s (Mathew et al., 1979; Sims et al., 1978). A possible explanation is that several factors contribute to the variability of salivary Li concentrations, possibly difficulting the implementation of salivary measurements into clinical practice. These include $\mathrm{Li}$ ion concentration in blood, stimulation of salivary glands, co-occurring medical conditions or treatments (Spencer et al., 1990; Spring and Spirtes, 1969). For instance, alimentary aspects, concurrent use of drugs affecting kidney function or acute infective kidney conditions could represent an obstacle in the implementation of Li salivary measurements into clinical practice (Thomsen and Schou, 1999).

Several recent findings in the sampling and analytical methods, as well as the growing interest in free drug concentrations, provide a new impetus for salivary measurement for a quick and non-invasive monitoring of therapeutic levels of mood stabilizers treatments for BD patients. For this reason, alongside with $\mathrm{Li}$, we decided to investigate the possible salivary bioequivalence of VPA too, with an improved methodology that allowed for greater detectability. In the present study, no significant correlation between VPA salivary and serum concentrations was found. In previous studies, either strong or weak association for VPA levels in both serum and saliva were reported, so that a significant correlation of saliva levels with serum levels of VPA was deemed possible (Soldin, 1999) or not (Liu and Delgado, 1999; Patsalos et al., 2008; Patsalos and Berry, 2013), depending on the type of analytical approach used. It is possible that the poor association between VPA concentration in serum and saliva can be due to differences in the rate of metabolization of the drug and the heterogeneity of the population, similarly to what happens in epileptic populations (Dwivedi et al., 2015). In the Dwivedi and cols study, a significant correlation was found between saliva and free serum VPA concentration, but the analysis of free serum VPA (serum or saliva) requires specialized laboratory techniques that may not be available in many hospital laboratories, limiting this possibility due to cost-effectiveness considerations (Dwivedi et al., 2015).

4.1 Clinical applicability 
Based on the results of the present bioequivalence study, Li-treated, but not VPA-treated, patients could benefit from the easy and quick measurement represented by salivary measurements. The advantages of salivary measures are the rapid patient administration and the non-invasive nature of the method. Salivary measurement of $\mathrm{Li}$ can be an option for unscheduled concentration esteems, giving the clinician a more reliable measures of patients' true status of adherence to pharmacological treatment, at all ages, as compared to the routinely scheduled lab tests.

As previously said, patients affected by BD are at risk of poor treatment adherence. Interestingly, patients for whom Li and VPA are more effective in maintenance treatment, i.e. with manic predominant polarity (Carvalho et al., 2014; Colom et al., 2006; Popovic et al., 2012; Samalin et al., 2016) are also more often poorly adherent to treatments and usually have a worse course of illness (Martinez-Aran et al., 2009; Murru et al., 2013). Predominant polarity has relevant therapeutic implications and is likely to be included in future editions of DSM (Carvalho et al., 2015; Colom et al., 2015; Vieta, 2016).

It is widely known that individuals with high levels of needle fear who also have chronic health conditions requiring injections (e.g., diabetes or multiple sclerosis) represent a particularly vulnerable group requiring special attention to this aspect, in order to maximize adherence to their medical regimen and avoid negative health effects (Mohr et al., 2001; Wright et al., 2009). In addition to the aforementioned comfort of procedure, salivary measure may thus reduce potential resistance to treatment in those people with a needle fear, a condition which prevalence in the general population ranges from 3$4.5 \%$ (LeBeau et al., 2010) for clinically relevant phobia up to approximately $10 \%$ for diagnostic subthreshold fear (Taddio et al., 2012). This condition may be especially prevalent and play a major role in children/young adults attitude towards treatment, similarly to what observed in other chronic conditions (Cemeroglu et al., 2015).

\section{CONCLUSIONS}

Lithium, but not valproate, presents with a high correlation in salivary and serum measurement. This potentially opens the door to a routine clinical use of salivary measures that could grant, in appropriate subpopulations of patients affected by BD, a feasible, non-invasive method to help assessing adherence to treatment. In future, studies assessing the non-inferiority of Li salivary concentration measurements, compared to standard serum dosing procedures, should be performed, in order to understand the possible application to everyday practice. 


\section{REFERENCES}

al Za'abi, M., Deleu, D., Batchelor, C., 2003. Salivary free concentrations of anti-epileptic drugs: an evaluation in a routine clinical setting. Acta Neurol. Belg. 103, 19-23.

American Psychiatric Association, 2000. DSM-IV-TR: Diagnostic and Statistical Manual of Mental Disorders, IV Edition. ed. APA, Washington DC. doi:10.1001/jama.1994.03520100096046

Ben-Aryeh, H., Laor, R., Szargel, R., Gutman, D., Naon, H., Pascal, M., Hefetz, A., 1984. Saliva for monitoring of patients with primary affective disorders. Isr. J. Med. Sci. 20, 197-201.

Bertholf, R.L., Savory, M.G., Winborne, K.H., Hundley, J.C., Plummer, G.M., Savory, J., 1988. Lithium determined in serum with an ion-selective electrode. Clin. Chem. 34, 1500-2.

Carvalho, A.F., McIntyre, R.S., Dimelis, D., Gonda, X., Berk, M., Nunes-Neto, P.R., Cha, D.S., Hyphantis, T.N., Angst, J., Fountoulakis, K.N., 2014. Predominant polarity as a course specifier for bipolar disorder: A systematic review. J. Affect. Disord. 163, 56-64. doi:10.1016/j.jad.2014.03.035

Carvalho, A.F., Quevedo, J., McIntyre, R.S., Soeiro-de-Souza, M.G., Fountoulakis, K.N., Berk, M., Hyphantis, T.N., Vieta, E., 2015. Treatment Implications of Predominant Polarity and the Polarity Index: A Comprehensive Review. Int. J. Neuropsychopharmacol. 18, pyu079-pyu079. doi:10.1093/ijnp/pyu079

Cemeroglu, A.P., Can, A., Davis, A.T., Cemeroglu, O., Kleis, L., Daniel, M.S., Bustraan, J., Koehler, T.J., 2015. Fear of needles in children with type 1 diabetes mellitus on multiple daily injections and continuous subcutaneous insulin infusion. Endocr. Pract. 21, 46-53. doi:10.4158/EP14252.OR

Colom, F., Vieta, E., Daban, C., Pacchiarotti, I., Sánchez-Moreno, J., 2006. Clinical and therapeutic implications of predominant polarity in bipolar disorder. J. Affect. Disord. 93, 13-7. doi:10.1016/j.jad.2006.01.032

Colom, F., Vieta, E., Martínez-Arán, A., Reinares, M., Benabarre, A., Gastó, C., 2000. Clinical factors associated with treatment noncompliance in euthymic bipolar patients. J. Clin. Psychiatry 61, 54955.

Colom, F., Vieta, E., Suppes, T., 2015. Predominant polarity in bipolar disorders: refining or redefining diagnosis? Acta Psychiatr. Scand. 132, 324-6. doi:10.1111/acps.12503

Danhof, M., Breimer, D.D.,. Therapeutic drug monitoring in saliva. Clin. Pharmacokinet. 3, 39-57.

Dwivedi, R., Gupta, Y.K., Singh, M., Joshi, R., Tiwari, P., Kaleekal, T., Tripathi, M., 2015. Correlation of saliva and serum free valproic acid concentrations in persons with epilepsy. Seizure 25, 187-90. doi:10.1016/j.seizure.2014.10.010

El-Mallakh, R.S., Linder, M., Valdes, R., Looney, S., 2004. Dialysis of saliva improves accuracy of saliva lithium determinations. Bipolar Disord. 6, 87-9.

Geddes, J.R., Miklowitz, D.J., 2013. Treatment of bipolar disorder. Lancet (London, England) 381, 167282. doi:10.1016/S0140-6736(13)60857-0

Gianfrancesco, F.D., Sajatovic, M., Rajagopalan, K., Wang, R.-H., 2008. Antipsychotic treatment adherence and associated mental health care use among individuals with bipolar disorder. Clin. Ther. 30, 1358-74.

González-Pinto, A., Reed, C., Novick, D., Bertsch, J., Haro, J.M., 2010. Assessment of medication adherence in a cohort of patients with bipolar disorder. Pharmacopsychiatry 43, 263-70. 


\section{doi:10.1055/s-0030-1263169}

Grande, I., Berk, M., Birmaher, B., Vieta, E., 2015. Bipolar disorder. Lancet (London, England). doi:10.1016/S0140-6736(15)00241-X

Johnson, R.E., McFarland, B.H., 1996. Lithium use and discontinuation in a health maintenance organization. Am. J. Psychiatry 153, 993-1000.

Keck, P.E., McElroy, S.L., Strakowski, S.M., Bourne, M.L., West, S.A., 1997. Compliance with maintenance treatment in bipolar disorder. Psychopharmacol. Bull. 33, 87-91.

Keller, M.B., Lavori, P.W., Coryell, W., Endicott, J., Mueller, T.I., 1993. Bipolar I: a five-year prospective follow-up. J. Nerv. Ment. Dis. 181, 238-45.

LeBeau, R.T., Glenn, D., Liao, B., Wittchen, H.-U., Beesdo-Baum, K., Ollendick, T., Craske, M.G., 2010. Specific phobia: a review of DSM-IV specific phobia and preliminary recommendations for DSM-V. Depress. Anxiety 27, 148-67. doi:10.1002/da.20655

Leppi, T.J., Spicer, S.S., 1966. The histochemistry of mucins in certain primate salivary glands. Am. J. Anat. 118, 833-59. doi:10.1002/aja.1001180310

Lew, K.H., Chang, E.Y., Rajagopalan, K., Knoth, R.L., 2006. The effect of medication adherence on health care utilization in bipolar disorder. Manag. Care Interface 19, 41-6.

Liu, H., Delgado, M.R., 1999. Therapeutic drug concentration monitoring using saliva samples. Focus on anticonvulsants. Clin. Pharmacokinet. 36, 453-70. doi:10.2165/00003088-199936060-00006

Mangoni, A.A., Jackson, S.H.D., 2004. Age-related changes in pharmacokinetics and pharmacodynamics: basic principles and practical applications. Br. J. Clin. Pharmacol. 57, 6-14.

Martinez-Aran, A., Scott, J., Colom, F., Torrent, C., Tabares-Seisdedos, R., Daban, C., Leboyer, M., Henry, C., Goodwin, G.M., Gonzalez-Pinto, A., Cruz, N., Sanchez-Moreno, J., Vieta, E., 2009. Treatment nonadherence and neurocognitive impairment in bipolar disorder. J. Clin. Psychiatry 70, 1017-23. doi:10.4088/JCP.08m04408

Mathew, R.J., Claghorn, J.L., Fenimore, D., Davis, C., Weinman, M., 1979. Saliva lithium and lithium therapy. Am. J. Psychiatry 136, 851. doi:10.1176/ajp.136.6.851

Merikangas, K.R., Jin, R., He, J.-P., Kessler, R.C., Lee, S., Sampson, N.A., Viana, M.C., Andrade, L.H., Hu, C., Karam, E.G., Ladea, M., Medina-Mora, M.E., Ono, Y., Posada-Villa, J., Sagar, R., Wells, J.E., Zarkov, Z., 2011. Prevalence and correlates of bipolar spectrum disorder in the world mental health survey initiative. Arch. Gen. Psychiatry 68, 241-51. doi:10.1001/archgenpsychiatry.2011.12

Mohr, D.C., Boudewyn, A.C., Likosky, W., Levine, E., Goodkin, D.E., 2001. Injectable medication for the treatment of multiple sclerosis: the influence of self-efficacy expectations and injection anxiety on adherence and ability to self-inject. Ann. Behav. Med. 23, 125-32.

Montoya, A., Pérez Sánchez Toledo, J., Gilaberte, I., González-Pinto, A., Haro, J.M., Vieta, E., Tohen, M., 2007. Patterns of drug treatment for manic episode in the clinical practice. Outcomes of the Spanish sample in the EMBLEM Study. Actas españolas Psiquiatr. 35, 315-22.

Moody, J.P., 1999. Biologic variation of serum and salivary lithium. Ther. Drug Monit. 21, 97-101.

Mucklow, J.C., Bacon, C.J., Hierons, A.M., Webb, J.K., Rawlins, M.D., 1981. Monitoring of phenobarbitone and phenytoin therapy in small children by salivary samples. Ther. Drug Monit. 3, 275-7. 
Murru, A., Pacchiarotti, I., Amann, B.L., Nivoli, A.M.A., Vieta, E., Colom, F., 2013. Treatment adherence in bipolar i and schizoaffective disorder, bipolar type. J. Affect. Disord. 151, 1003-1008.

Murru, A., Popovic, D., Pacchiarotti, I., Hidalgo, D., León-Caballero, J., Vieta, E., 2015. Management of Adverse Effects of Mood Stabilizers. Curr. Psychiatry Rep. 17, 603. doi:10.1007/s11920-015-0603Z

Neu, C., DiMascio, A., Williams, D., 1975. Saliva lithium levels: clinical applications. Am. J. Psychiatry 132, 66-8. doi:10.1176/ajp.132.1.66

NICE, 2014. Bipolar disorder: the assessment and management of bipolar disorder in adults, children and young people in primary and secondary care, NICE guide. ed. National Collaborating Centre for Mental Health, London.

Nivoli, A.M.A., Murru, A., Vieta, E., 2010. Lithium: Still a cornerstone in the long-term treatment in bipolar disorder? Neuropsychobiology.

Pacchiarotti, I., León-Caballero, J., Murru, A., Verdolini, N., Furio, M.A., Pancheri, C., Valentí, M., Samalin, L., Roigé, E.S., González-Pinto, A., Montes, J.M., Benabarre, A., Crespo, J.M., de Dios Perrino, C., Goikolea, J.M., Gutiérrez-Rojas, L., Carvalho, A.F., Vieta, E., 2016. Mood stabilizers and antipsychotics during breastfeeding: Focus on bipolar disorder. Eur. Neuropsychopharmacol. 26, 1562-1578. doi:10.1016/j.euroneuro.2016.08.008

Patsalos, P.N., Berry, D.J., 2013. Therapeutic Drug Monitoring of Antiepileptic Drugs by Use of Saliva. Ther. Drug Monit. 35, 4-29. doi:10.1097/FTD.0b013e31827c11e7

Patsalos, P.N., Berry, D.J., Bourgeois, B.F.D., Cloyd, J.C., Glauser, T.A., Johannessen, S.I., Leppik, I.E., Tomson, T., Perucca, E., 2008. Antiepileptic drugs--best practice guidelines for therapeutic drug monitoring: a position paper by the subcommission on therapeutic drug monitoring, ILAE Commission on Therapeutic Strategies. Epilepsia 49, 1239-76. doi:10.1111/j.15281167.2008.01561.x

Pérez-Castelló, I., Mangas-Sanjuan, V., González-García, I., Gonzalez-Alvarez, I., Bermejo, M., MarcoGarbayo, J.L., Trocóniz, I.F., 2016. Population pharmacokinetic model of lithium and drug compliance assessment. Eur. Neuropsychopharmacol. 26, 1868-1876. doi:10.1016/j.euroneuro.2016.11.005

Popovic, D., Reinares, M., Goikolea, J.M., Bonnin, C.M., Gonzalez-Pinto, A., Vieta, E., 2012. Polarity index of pharmacological agents used for maintenance treatment of bipolar disorder. Eur. Neuropsychopharmacol. 22, 339-46. doi:10.1016/j.euroneuro.2011.09.008

Sajatovic, M., Ignacio, R. V, West, J.A., Cassidy, K.A., Safavi, R., Kilbourne, A.M., Blow, F.C., 2009. Predictors of nonadherence among individuals with bipolar disorder receiving treatment in a community mental health clinic. Compr. Psychiatry 50, 100-7. doi:10.1016/j.comppsych.2008.06.008

Samalin, L., Murru, A., Vieta, E., 2016. Management of inter-episodic periods in patients with bipolar disorder. Expert Rev. Neurother. 1-12. doi:10.1080/14737175.2016.1176530

Shetty, S.J., Desai, P.B., Patil, N.M., Nayak, R.B., 2012. Relationship between serum lithium, salivary lithium, and urinary lithium in patients on lithium therapy. Biol. Trace Elem. Res. 147, 59-62. doi:10.1007/s12011-011-9295-3 
Sims, A., White, A.C., Garvey, K., 1978. Problems associated with the analysis and interpretation of saliva lithium. Br. J. Psychiatry 132, 152-4.

Soldin, S.J., 1999. Free drug measurements. When and why? An overview. Arch. Pathol. Lab. Med. 123, 822-3. doi:10.1043/0003-9985(1999)123<0822:FDM>2.0.CO;2

Spencer, E.K., Campbell, M., Adams, P., Perry, R., Choroco, M.C., Padron-Gayol, M., Small, A.M., 1990. Saliva and serum lithium monitoring in hospitalized children. Psychopharmacol. Bull. 26, 239-43.

Spring, K.R., Spirtes, M.A., 1969. Salivary excretion of lithium. I. Human parotid and submaxillary secretions. J. Dent. Res. 48, 546-9.

Taddio, A., Ipp, M., Thivakaran, S., Jamal, A., Parikh, C., Smart, S., Sovran, J., Stphens, D., Katz, J., 2012. Survey of the prevalence of immunization non-compliance due to needle fears in children and adults. Vaccine 30, 4807-4812.

Thomsen, K., Schou, M., 1999. Avoidance of lithium intoxication: advice based on knowledge about the renal lithium clearance under various circumstances. Pharmacopsychiatry 32, 83-6. doi:10.1055/s2007-979199

Tondo, L., Abramowicz, M., Alda, M., Bauer, M., Bocchetta, A., Bolzani, L., Calkin, C. V., Chillotti, C., Hidalgo-Mazzei, D., Manchia, M., Müller-Oerlinghausen, B., Murru, A., Perugi, G., Pinna, M., Quaranta, G., Reginaldi, D., Reif, A., Ritter, P., Rybakowski, J.K., Saiger, D., Sani, G., Selle, V., Stamm, T., Vázquez, G.H., Veeh, J., Vieta, E., Baldessarini, R.J., 2017. Long-term lithium treatment in bipolar disorder: effects on glomerular filtration rate and other metabolic parameters. Int. J. Bipolar Disord. 5, 27. doi:10.1186/s40345-017-0096-2

Tonic-Ribarska, J., Haxhiu, A., Sterjev, Z., Kiteva, G., Suturkova, L., Trajkovic-Jolevska, S., 2012. Development and validation of a bioanalytical LC-UV method with solid-phase extraction for determination of valproic acid in saliva. Acta Pharm. 62, 211-20. doi:10.2478/v10007-012-0015-0

Velligan, D.I., Weiden, P.J., Sajatovic, M., Scott, J., Carpenter, D., Ross, R., Docherty, J.P., 2009. The expert consensus guideline series: adherence problems in patients with serious and persistent mental illness. J. Clin. Psychiatry 70 Suppl 4, 1-46-8.

Verghese, A., Indrani, N., Kuruvilla, K., Hill, P.G., 1977. Usefulness of saliva lithium estimation. Br. J. Psychiatry 130, 148-50.

Vieta, E., 2016. DSM-5.1. Acta Psychiatr. Scand. 134, 187-8. doi:10.1111/acps.12624

Wright, S., Yelland, M., Heathcote, K., Ng, S.-K., Wright, G., 2009. Fear of needles--nature and prevalence in general practice. Aust. Fam. Physician 38, 172-6.

Yatham, L.N., Kennedy, S.H., Parikh, S. V, Schaffer, A., Beaulieu, S., Alda, M., O’Donovan, C., Macqueen, G., McIntyre, R.S., Sharma, V., Ravindran, A., Young, L.T., Milev, R., Bond, D.J., Frey, B.N., Goldstein, B.I., Lafer, B., Birmaher, B., Ha, K., Nolen, W.A., Berk, M., 2013. Canadian Network for Mood and Anxiety Treatments (CANMAT) and International Society for Bipolar Disorders (ISBD) collaborative update of CANMAT guidelines for the management of patients with bipolar disorder: update 2013. Bipolar Disord. 15, 1-44. doi:10.1111/bdi.12025 


\section{Acknowledgments and Funding Sources}

Dr. Francesc Colom would like acknowledge FIS- Instituto de Salud Carlos III (PI 12/00910) Miguel Servet II (MSII14/00030), 2014_SGR_398 (Secretaria d'Universitats i Recerca del Departament d'Economia i Coneixement, Generalitat de Catalunya).

Prof. Vieta would like to acknowledge PI 12/00912 (Spanish Ministry of Economy and Competitiveness), PN 2008-2011 (Instituto de Salud Carlos III, Subdirección General de Evaluación y Fomento de la Investigación; Fondo Europeo de Desarrollo Regional. Unión Europea, "Una manera de hacer Europa"), 2014_SGR_398 (Secretaria d'Universitats i Recerca del Departament d'Economia i Coneixement Generalitat de Catalunya) 